\title{
The NHEJ Repair of DNA Double Strand Breaks in Physcomitrella patens Depends on the Kleisin NSE4 of the SMC5/6 Complex.
}

\author{
Radka Vágnerová, Marcela Holá, Karel J. Angelis
}

The Czech Academy of Sciences, Institute of Experimental Botany, Na Karlovce 1, CZ16000, Prague, Czech Republic (karel.angelis@gmail.com)

\begin{abstract}
Structural maintenance of chromosomes (SMC) complexes are involve in cohesion, condensation and maintenance of genome stability. Based on the sensitivity of mutants to genotoxic stress the SMC5/6 complex is thought to play imminent role in DNA stabilization during repair by encircling DNA at the site of lesion by bridging the heteroduplex of SMC5 and SMC6 by non SMC kleisin components NSE1, 3 and 4. In this study, we tested how formation of the SMC5/6 circular structure affects mutant sensitivity to genotoxic stress, kinetics of DSB repair and insertion mutagenesis. In the moss Physcomitrella patens SMC6 and NSE4 are essential single copy genes and this is why we used blocking of transcription to reveal their mutated phenotype. Even slight attenuation of transcription by dCas9 binding was enough to obtain stable lines with DSB repair defect and specific bleomycin sensitivity. Whereas survival after bleomycin or MMS treatment fully depends on active SMC6, NSE4 has little or negligible effect. We conclude that whereas circularization of SMC5/6 provided by the kleisin NSE4 is indispensable for the immediate NHEJ DSB repair response, other functions associated with SMC5/6 complex are critical to survive DNA damage.
\end{abstract}

Keywords: SMC5/6; NSE4 kleisin; dCas9; DNA repair; gene targeting; comet assay

\section{INTRODUCTION}

Plants as sessile organisms developed several strategies to protect integrity of their genomes against various environmental issues including genotoxic stress. The bryophyte

Physcomitrella patens ( $P$. patens) stands aside from other plants with high frequency of homologous recombination (HR) due to its remarkable ability to integrate transgenes at predefined loci through homologous recombination-mediated gene targeting (GT) (Kamisugi et al. 2016). Experimentally, P. patens is highly amenable to analysis and manipulation e.g. for study of DNA damage and repair by comet assay (Angelis et al. 2015). P. patens spore develops into a haploid filamentous structure called protonema, composed of two types of cells - chloronema and caulonema. With respect to in vitro cultivation conditions in basal medium enriched with ammonium tartrate (Knight et al. 2002) protonema is exclusively composed of chloronemal cells accumulated at the G2/M transition. This unique tissuespecific cell cycle arrest is thought to be behind uniquely high rate of HR in $P$. patens nuclear DNA (Schween et al. 2003). Protonema filaments grow exclusively by tip growth of their apical cells (Menand et al. 2007). The advantage of using filamentous P. patens is that it can be employed either as a culture of dividing cells approximated by 3-7 cell fragments with average $50 \%$ of apical cells obtained from protonemal lawns by extensive shearing or as differentiated tissue from 7-days grown protonemata with only $5 \%$ of apical cells. Either culture exhibits different kinetics of DNA repair (Goffova et al. 2019; Kamisugi et al. 2012). APT (adenine phosphoribosyltransferase) is an enzyme of the purine salvage pathway that converts adenine into AMP and its loss of function generates plants resistant to adenine analogues e.g. 2-fluoroadenine (2-FA) (Gaillard et al. 1998). Mutational inactivation can be 
used as selectable marker as well as analysis of mutations in APT locus on nucleotide level (Trouiller et al. 2007; Trouiller et al. 2006).

The SMC6 ( structural maintenance of chromosome) protein is component of highly conserved SMC5/6 complex that is composed of SMC5 and SMC6 heterodimer and 6 nonSMC elements NSE1-6. Similarly to the other SMC complexes of cohesin and condensin, SMC5-SMC6 heterodimer together with NSE4 klesin-bridge form circular structure capable of entrapping DNA (Hassler et al. 2018). The NSE1 and NSE3 subunits regulate directly or indirectly the ring shape and functions (Vondrova et al. 2019). This conserved core of the SMC5/6 complex is indispensable for all its functions including essential processes like DNA replication and repair (Diaz and Pecinka 2018; Palecek 2018). NSE4 and SMC6 are essential genes in yeast and mammals and their knockout is lethal (Hu et al. 2005; Ju et al. 2013; Onoda et al. 2004). The Arabidopsis orthologue AtSMC6b (MIM) was shown to interfere with DSBs repair by eliminating its rapid phase (Kozak et al. 2009). Because the haploid state of the $P$. patens protonema and indispensability of the SMC5/6 complex do not allow complete depletion of any of the complex subunits we used attenuated expression approach by specific binding of catalytically dead Cas9 (dCas9) without endonucleolytic activity to desired gene and generate mutants with attenuated transcription. Such mutant plants are still viable, growing and manifesting DNA repair phenotype.

\section{MATERIALS AND METHODS}

\section{Plant Material and Cultivation}

The WT Physcomitrella patens (accession (Hedw.) B.S.G. 'Gransden2004') (Knight et al. 2002), the ppku70 and pprad51-1-2 double mutant (Schaefer et al. 2010) were generated by D. G. Schaefer, Neuchatel University, Switzerland, and F. Nogué, INRA, Paris, France, and kindly provided by F. Nogué. All strains were cultured as 'spot inocula' on BCD agar medium supplemented with $1 \mathrm{mM} \mathrm{CaCl} 2$ and $5 \mathrm{mM}$ ammonium tartrate (BCDAT medium), or as lawns of protonema filaments by subculture of homogenized tissue on BCDAT agar overlaid with cellophane in growth chambers with $18 / 6 \mathrm{~h}$ day/night cycle at $22 / 18^{\circ} \mathrm{C} \mathrm{(Knight}$ et al. 2002).

One-day-old protonema tissue (1d) for repair experiments was prepared from 1-week-old tissue (7d) scraped from plate, suspended in $8 \mathrm{ml}$ of BCDAT medium, sheared at $10000 \mathrm{rpm}$ for two, 1-min cycles by a T25 homogenizer (IKA, Germany) and let to recover for $24 \mathrm{~h}$ in cultivation chamber with gentle shaking at $100 \mathrm{rpm}$. This treatment yielded a suspension of 3-5 cell protonemata filaments, which readily settle for recovery.

Generation and Analysis of PpNSE4 and PpSMC6 Mutants

The vectors for attenuation of PpNSE4 and PpSMC6 expression were constructed as expression cassette of dCas9 driven by maize ubiquitin promoter, NPTII selection cassette and specific guide RNA (sgRNA). Construction, transformation and validation of mutated lines is described in Supplementary Methods S1.

Three stable transformants of ppnse 4 and ppsmc 6 were randomly picked and analyzed by quantitative RT-PCR (qRT-PCR) for NSE4 or SMC6 expression (Fig. 1b). Total RNA was isolated from 7 days old protonemata with RNeasy Plant Mini Kit (Qiagen), treated with DNaseI (DNA-free ${ }^{\mathrm{TM}}$ DNA Removal Kit, Thermo Fisher Scientific) and reverse transcribed using qPCRBIO cDNA Synthesis Kit (PCR Biosystems). Diluted cDNA reaction mixtures were used for qRT-PCR analysis using the qPCRBIO SyGreen Mix Lo-ROX (PCR Biosystems) in Statagene-MX3005P. Analysis was performed for three biological replicas 
(independently cultivated tissue) and in two technical replicates with Clathrin adapter complex subunit CAP-50 (Pp3c27_2250V3.1) as a reference gene (Kamisugi et al. 2016). The relative transcription of NSE4 and SMC6 was calculated by the $\triangle \triangle C t$ method (Pfaffl 2004).

\section{Mutagens, Treatments and Sensitivity Assay}

Sensitivity to DNA damage was measured after treatment either with freshly prepared solutions of bleomycin sulphate supplied as Bleomedac inj. (Medac, Hamburg, Germany), or methyl methanesulfonate (MMS) (Sigma-Aldrich) in BCDAT medium. Protonemata were dispersed in liquid BCDAT medium containing bleomycin or MMS and treated for $1 \mathrm{~h}$. After treatment and washing recovered protonemata were inoculated as eight explants per quadrant of a Petri dish with drug-free BCDAT agar without cellophane overlay and left to grow. UVC irradiation was performed in a Hoefer UVC 500 crosslinker at $254 \mathrm{~nm}$ by irradiating $P$. patens lines spotted as explants on a Petri dish. Irradiation and the following steps were performed for $24 \mathrm{~h}$ in the dark or under red illumination to block photolyases. Treatment was assessed after 3 weeks by weighing explants. The fresh weight of the treated explants was normalized to the fresh weight of untreated explants of the same line and plotted as $\%$ of 'Relative fresh weight'. In every experiment, the treatment was carried in duplicate and experiments were repeated two or three times and statistically analyzed by Student t-test.

\section{Single-Cell Gel Electrophoresis (Comet) Assay}

Repair kinetics was estimated in $1 \mathrm{~d}$ and $7 \mathrm{~d}$ protonemata after bleomycin or MMS treatment. Tissue was either flash-frozen in liquid $\mathrm{N}_{2}$ (repair $\mathrm{t}=0$ ) or left to recover in liquid BCDAT medium for the indicated repair times and then frozen. DSBs after bleomycin treatment were detected by a comet assay using neutral N/N protocol whereas SSBs after MMS treatment were detected with A/N protocol, which includes after lysis of nuclei a treatment with alkali to reveal breaks by unwinding DNA double helix as described in (Angelis et al. 1999; Hola et al. 2013). Comets were stained with SYBR Gold (Molecular Probes/Invitrogen) and viewed in epifluorescence with a Nikon Eclipse 800 microscope and evaluated by the LUCIA Comet cytogenetic software (LIM Inc., Prague, Czech Republic).

The fraction of DNA in comet tails ( $\%$ tail-DNA) was used as a measure of DNA damage. In each experiment, the \% tail-DNA was measured at seven time points: $0,3,5,10,20,60$ and $180 \mathrm{~min}$ after the treatment and in control tissue without treatment. Measurements obtained in at least three independent experiments and totaling at least 300 comets analyzed per experimental point were plotted as \% of remaining damage and statistically analyzed by Student t-test.

\section{Gene Targeting Assay}

GT efficiencies were assessed after transformation with the APT based targeting construct pKA255 (Fig. 4). Vector pKA225 contains 35S-Hygromycin marker (HygroR) flanked by 775bps 5'-homology fragment of APT locus (Pp3c8_16590V3.1) (position -766 - 9 across the start codon) and 622 bps 3 '-homology fragment $(65 \overline{8}-1280)$ that were amplified from moss genomic DNA by PCR. Linear targeting fragment was amplified by PCR and delivered by PEG mediated transformation to WT and ppnse4-4 and ppsmc6-3 protoplasts (Liu and Vidali 2011). Transformed protoplasts of each line were spread on four Petri dishes with BCDAT medium overlaid with cellophane disc. Regenerating protoplasts was counted after 5 days on each Petri dish and then transferred to new Petri dish with BCDAT medium supplemented 
with $5 \mu \mathrm{M}$ 2-FA. Successful transformation was followed by direct counting of 2-FA resistant $\left(2-\mathrm{FA}^{\mathrm{R}}\right)$ colonies after 3 weeks. Frequency of GT is expressed as the ratio between the number of $2-\mathrm{FA}^{\mathrm{R}}$ colonies and the number of regenerating protoplasts. Experiments were repeated three times and statistically analyzed using the Fisher exact test.

In recovered 2-FA ${ }^{\mathrm{R}}$ clones integration of pKA255 was checked by PCR in six randomly picked 2-FA ${ }^{\mathrm{R}}$ clones of each line with external gene primers 868 and 727 and selection cassette primers 1023 and 1024 and integration borders were confirmed by sequencing with primers $862,857,1259$ and 1258 (Supplementary Table S1).

Statistical analysis.

Three biological repeats were conducted for each experiment. Statistical significance between two samples was analyzed by two-tailed Student's $t$ test.

\section{RESULTS and DISCUSSION}

Generation and Analysis of ppnse4 and ppsmc6 Mutants

Based on protein sequence of $A$. thaliana search in $P$. patens genome (Phytozome v12.1) we identified single copy locus of NSE4 (Pp3c27_130V3.1) and of SMC6 (Pp3c11_11190V3.1). In P. patens NSE4 and SMC6 are essential genes and their deletion is lethal (unpublished observation), nevertheless it is possible to obtain stable dCas9_sgRNA transformants with targeted NSE4 promotor and SMC6 hinge region (Fig. 1a). It was not possible to recover any stable dCas9 transformant with targeted $S M C 6$ promotor or ATPase Walker A region.

Three rescued lines of NSE4- (ppnse4-4,5 and 6) and of SMC6- (ppsmc6-1,2,3) were analyzed for remaining expression by qRT-PCR (Fig. 1b). In this study we use ppnse $4-4$ with $15 \%$ and ppsmc6-3 with 40\% reduction of transcription, both the highest values in rescued lines.

Phenotype of ppnse4-4 though growing slightly slower do not manifest any obvious deviation from WT, whereas ppsmc6-3 growth is reduced, and plants do not develop gametophores (Fig. 1c).

The attenuation was repeatedly confirmed in both lines after several months. 
(a)

(b)
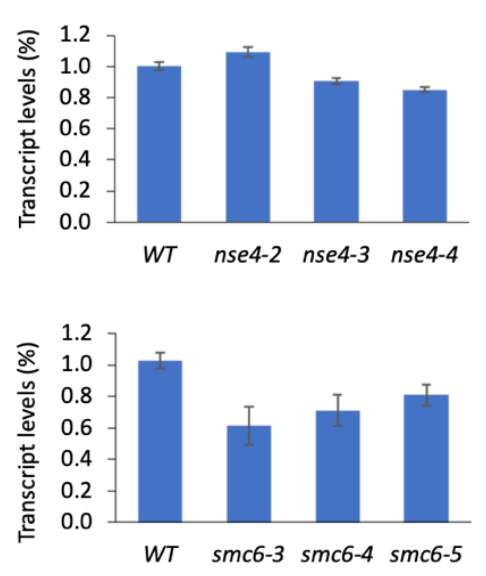

NSE4 (Pp3c27_130)

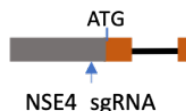

$(-165)$

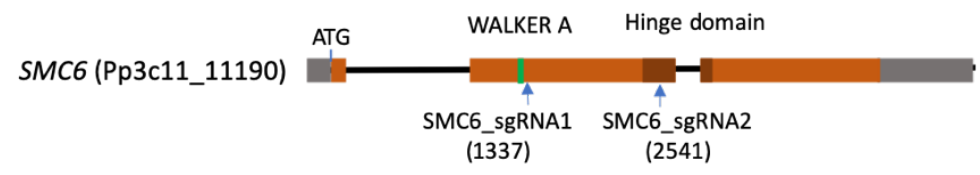

(c)

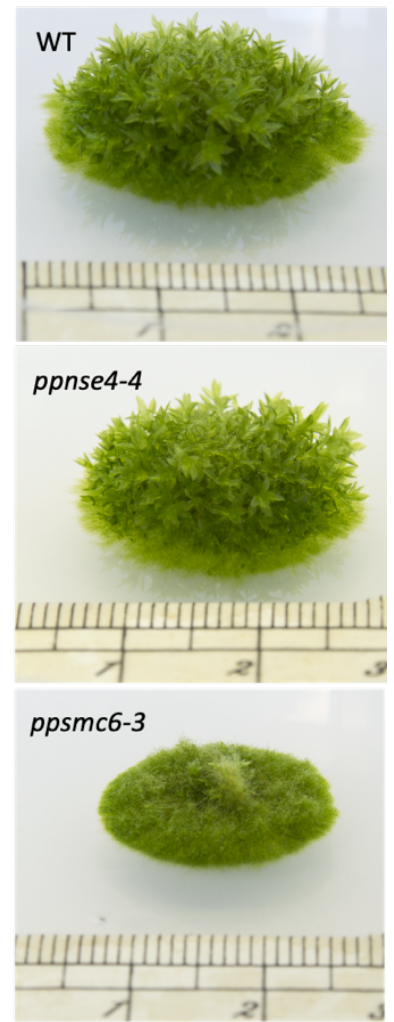

FIG. 1 Characterization of ppnse 4 and ppsmc 6 mutants. (a) Structure of the WT, PpNSE4, and PpSMC6 loci with indication of dCas9 binding. The brown boxes represent exons and the gray's UTR regions. (b) qRT-PCR analysis of NSE4 (upper) and SMC6 (lower) transcripts in WT and mutant plants. Primers are listed in Supplementary Table S1. (c) Morphology of colonies of WT and of ppnes 4-4 and ppsmc6-3 mutants. The picture was taken after 1.5 month of growth.

\section{Recovery from Bleomycin and MMS Treatment Depends on PpSMC6}

To characterize defects in ppnse4-4, smc6-3, ppku70 and pprad51-1-2 mutants we compared their growth-responses to lesions like DSBs induced by radiomimetic bleomycin, small alkylation adducts induced by MMS and DNA helix distortion by photo adducts induced by UVC irradiation. These lesions represent blocks for DNA replication and are removed, repair or bypassed by various error-free as well as error-prone pathways. The sensitivity was tested after acute treatment for $1 \mathrm{~h}$ as the ability of the tissue to recover.

In response to the treatment with bleomycin or MMS, sensitivities of ppnse4-4 and ppsmc6-3 substantially differed, both in cultures with dividing (1d) as well as differentiated (7d) cells. Whereas the sensitivity of ppnse4-4 was very similar to WT, ppsmc6-3 was extremely vulnerable (Fig. 2a,b,d,e). After exposure to $20 \mu \mathrm{g}$ bleomycin $/ \mathrm{ml}$ and $30 \mathrm{mM}$ MMS ppsmc6-3 explants did not recover at all, while WT and ppnse4-4 still retain about 50\%, and in $1 \mathrm{~d}$ culture treated with $30 \mathrm{mM}$ MMS even 100\% viability (Fig. 2b).

The response to bleomycin and MMS suggests that formation of circular form of SMC5/6 complex is not inevitably necessary to offset toxic effect of DNA lesions and so enable cell survival, but availability of complex itself and additional associated activities like SUMOylation and other so far undisclosed functions are critical and essential for survival. 
Response to UVC-irradiation does not give clear picture on the role of NSE4 and SMC6 in the response to induced lesions, nevertheless ppsmc6-3 remain the most vulnerable in dividing as well as differentiated tissue.
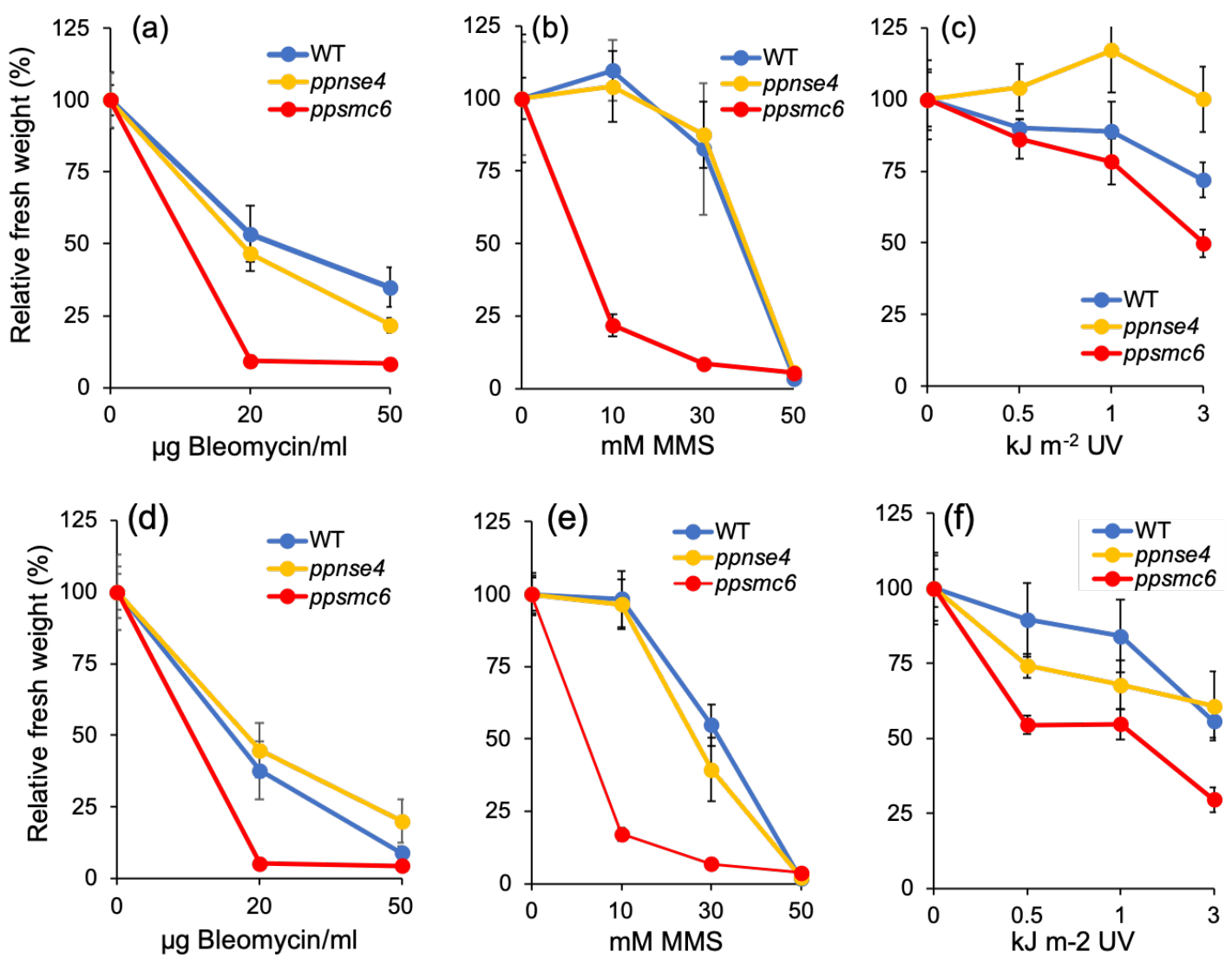

FIG. 2 Growth-responses of $1 \mathrm{~d}(\mathrm{a}, \mathrm{b}, \mathrm{c})$ and $7 \mathrm{~d}(\mathrm{~d}, \mathrm{e}, \mathrm{f})$ protonemata of $P$. patens $\mathrm{WT}$, nse $4-4$ and smc6-3 to $1 \mathrm{~h}$ treatment with bleomycin (a, d), MMS (b, e) or UVC-irradiation (c, f). After treatment, the explants were incubated on drug-free BCDAT medium under standard growth conditions for 3 weeks. For each experimental point the weight of treated plants collected from two replicas was normalized to the weight of untreated plants and plotted as relative fresh weight, which was set as a default to 100. Error bars indicate SD.

DSB Repair is Impaired in $n s e 4$ and smc6

DSB repair is severely inhibited in both ppnse 4-4 and ppsmc6-3 lines where both share identical repair kinetic in dividing (1d) as well as differentiated (7d) cells (Fig. 3a,c). In this respect they closely parallel previously reported repair defect in A. thaliana mutant atmim (atsmc6b) (Kozak et al. 2009), when SMC6b was suggested to participate in NHEJ due to absence of repair rapid phase that is assumed to represent NHEJ repair (Goodarzi et al. 2010). To ascertain the repair pathway where SMC6 is involved, we compared ppnse4-4 and ppsmc6-3 with cNHEJ mutant ppku70 and HR mutant pprad51-1-2. Statistical analysis showed that actually their repair kinetic differs from both, pprad51-1-2 $(1 \mathrm{~d} \mathrm{p}<0.01 ; 7 \mathrm{~d} \mathrm{p}<$ $0.05)$ and ppku70 ( $1 \mathrm{~d}$ and $7 \mathrm{~d} \mathrm{p}<0.05)$. This result supports conclusion from A. thaliana that SMC5/6 is involved in tethering DNA strands during alternative NHEJ, distinct from cNHEJ also in $P$. patens. For tethering DNA and participation in DSB repair bridging of ATPase heads of SMC5/6 heteroduplex by kleisin NSE4 is critical what is documented by the fact 
that weak, $15 \%$, attenuation of NSE4 transcription results in the same extent of repair deficit as $40 \%$ attenuation of SMC6 transcription.

MMS does not induce DSBs detectable by comet assay and repair of SSBs in WT and all mutants is completely abolished (Fig. 3b,d). This is in contrast with repair of bleomycin induced SSBs when in WT 50\% of breaks are already removed after 3h (Hola et al. 2013).
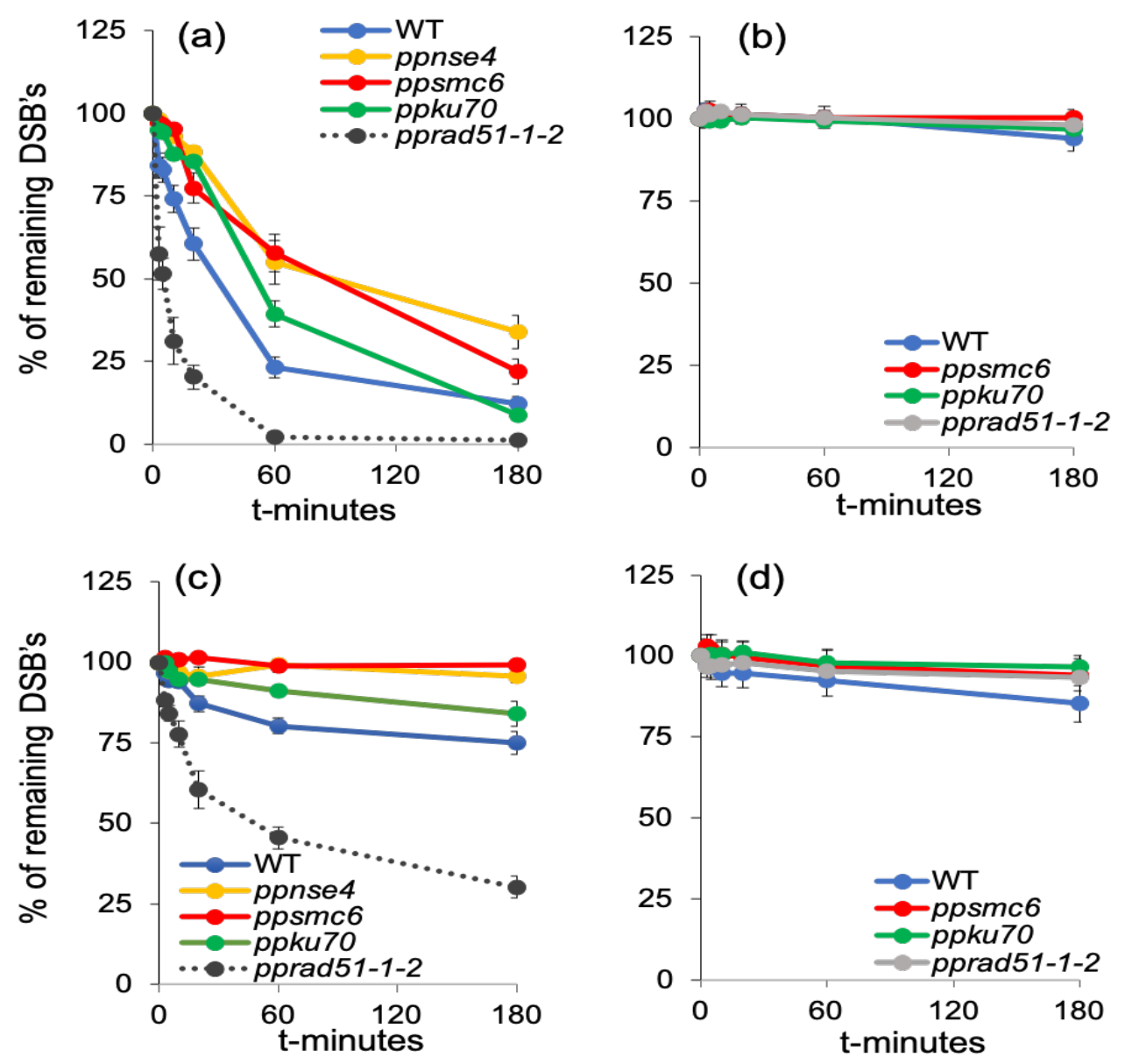

FIG. 3 DSB and SSB repair kinetics determined by comet assay in $1 \mathrm{~d}$ or $7 \mathrm{~d}$ tissue of WT (blue) and ppnse4,4 (orange), ppsmc6-3 (red), ppku70 (green) and pprad51-1-2 (gray) mutants. Data for pprad51-1-2 (a,c) are from (Goffova et al. 2019). Protonemata regenerated $1 \mathrm{~d}(\mathrm{a}, \mathrm{b})$ or $7 \mathrm{~d}(\mathrm{c}, \mathrm{d})$ after subculture were treated with $30 \mu \mathrm{g}$ bleomycin $/ \mathrm{ml}$ or $30 \mathrm{mM}$ MMS for $1 \mathrm{~h}$ and repair kinetics was measured as $\%$ of DSBs $(\mathrm{a}, \mathrm{c})$ or SSB $(\mathrm{b}, \mathrm{d})$ remaining after the $0,3,5,10,20,60$ and $180 \mathrm{~min}$ of repair recovery. Maximum damage is normalized as $100 \%$ at $\mathrm{t}=0$ for all lines. Error bars indicate SD.

Gene Targeting is Increased in the $n s e 4$ and smc6 Mutants

The GT vector pKA225 was designed to disrupt $P p A P T$ gene across the second exon site (229-339) previously identified as mutation hotspot (Hola et al. 2013; Hola et al. 2015). The GT rate was determined as the frequency of $2-\mathrm{FA}^{\mathrm{R}}$ plants amongst $10^{6}$ regenerating protoplasts (Fig. 4c). There is a statistically significant increase of GT rate after attenuation of NSE4 and SMC6. As evident from Fig. 4b the rate of GT is increased approximately $7 \mathrm{x}(\mathrm{p}<$ 0001) in ppnse4-4 and only $2 \mathrm{x}(\mathrm{p}<0.049)$ in ppsmc6-3 over WT. PCR analysis of 6 randomly selected $2-\mathrm{FA}^{\mathrm{R}}$ plants of each line revealed correct, full length integration. To 
assess accuracy of integration, insert borders were sequenced in two 2-FA ${ }^{\mathrm{R}}$ transformants of each line and all analyzed transformants had accurate insertion at both ends of insert without detectable mutation. This result supports above described finding that attenuation of SMC6 and particularly of NSE4 expression impairs error-prone NHEJ repair machinery of whatever mechanism is involved during integration and shift balance to error-free HR insertion.

(a)

APT (Pp3c8_16590)

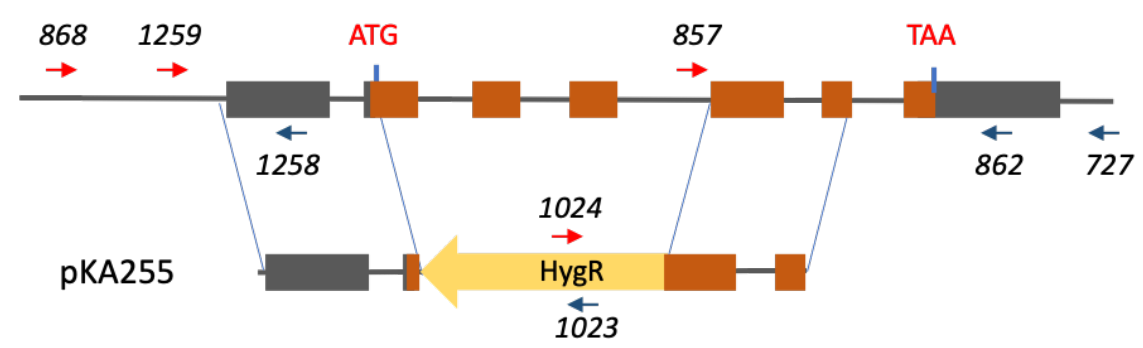

(b)

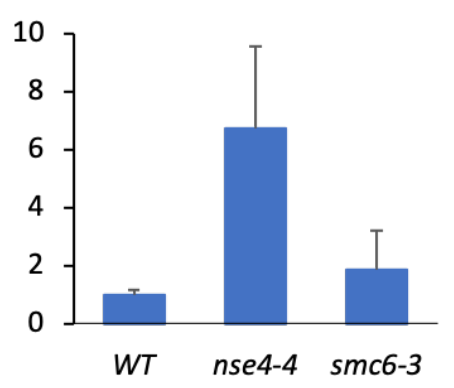

(c)

\begin{tabular}{|c|c|c|c|}
\hline Genotype & Regenerants & 2-FA resistent & Rate in $10^{-6}$ \\
\hline WT & 137922 & 16 & 116 \\
\hline nse4-4 & 92117 & $72(p<.0001)$ & 781 \\
\hline smc6-3 & 130033 & $28(p<.0449)$ & 215 \\
\hline
\end{tabular}

FIG. 4 GT assay. (a) Schematic drawing of APT locus and of targeting construct pKA255. The brown boxes represent exons and the gray's UTR regions. Arrows mark position of primers used to genotype and sequence the plants by PCR. (b) GT efficiency in ppnse4-4 and ppsmc6-3 in comparison to WT, which was set as a default to 1). (c) The rate of GT per $10^{6}$ protoplasts. Differences between WT and mutants were compared using Fisher's exact test.

\section{CONCLUSION}

In this study we used haploid $P$. patens cultivated on rich BCDAT medium as chloronema with cells arrested at G2/M border to study the role of SMC5/6 complex in DNA repair. SMC5/6 in circular form is indispensable for DSB repair presumably by NHEJ, but not cNHEJ pathway, what is illustrated by the same repair kinetic of the smc 6 and kleisin nse 4 mutants that is distinct from $k u 70$. Nevertheless, the repair dependent on NSE4 is not essential for cell survival after bleomycin and MMS treatment as the incurred damage can be offsetted by alternative repair mechanisms. The increased rate of GT shows that defect or elimination of NSE4 dependent pathway shifts equilibrium in favor of undisturbed HR mechanism. Still remain question, which functions and qualities of SMC5/6 enable survival of nse 4 after bleomycin or MMS treatment, but are disturbed in $s m c 6$, or whether the observed differences are just consequence of different degree of NSE4 and SMC6 expression.

\section{ACKNOWLEDGEMENTS}


The research was supported by the Czech Science Foundation (project 20-05095S) and by the Ministry of Education, Youth and Sports of the Czech Republic under the project COST (LTC17047).

\section{AUTHOR CONTRIBUTIONS}

$\mathrm{MH}$ and KJA designed the research, analyzed data and wrote the manuscript. RV, $\mathrm{MH}$ and KJA conducted the research.

\section{CONFLICT OF INTERESTS}

The authors declare no conflicts of interest.

\section{SUPPLEMENTARY MATERIALS}

The Supplementary Material for this article can be found online at:

\section{REFERENCES}

Angelis KJ, Dusinska M, Collins AR (1999) Single cell gel electrophoresis: detection of DNA damage at different levels of sensitivity Electrophoresis 20:2133-2138 doi:10.1002/(SICI)1522-2683(19990701)20:10<2133::AID-ELPS2133>3.0.CO;2-Q

Angelis KJ, Kozák J, Vágnerová R, Holá M (2015) DNA repair in plants studied by comet assay Frontiers in Genetics doi:10.3389/conf.fgene.2015.01.00067

Diaz M, Pecinka A (2018) Scaffolding for Repair: Understanding Molecular Functions of the SMC5/6 Complex Genes (Basel) 9 doi:10.3390/genes9010036

Gaillard C, Moffatt BA, Blacker M, Laloue M (1998) Male sterility associated with APRT deficiency in Arabidopsis thaliana results from a mutation in the gene APT1 Mol Gen Genet 257:348-353 doi:10.1007/s004380050656

Goffova I et al. (2019) Roles of RAD51 and RTEL1 in telomere and rDNA stability in Physcomitrella patens Plant J 98:1090-1105 doi:10.1111/tpj.14304

Goodarzi AA, Jeggo P, Lobrich M (2010) The influence of heterochromatin on DNA double strand break repair: Getting the strong, silent type to relax DNA Repair (Amst) 9:1273-1282 doi:10.1016/j.dnarep.2010.09.013

Hassler M, Shaltiel IA, Haering CH (2018) Towards a Unified Model of SMC Complex Function Curr Biol 28:R1266-R1281 doi:10.1016/j.cub.2018.08.034

Hola M, Kozak J, Vagnerova R, Angelis KJ (2013) Genotoxin induced mutagenesis in the model plant Physcomitrella patens Biomed Res Int 2013:535049 doi:10.1155/2013/535049

Hola M, Vagnerova R, Angelis KJ (2015) Mutagenesis during plant responses to UVB radiation Plant Physiol Biochem 93:29-33 doi:10.1016/j.plaphy.2014.12.013

$\mathrm{Hu}$ B et al. (2005) Qri2/Nse4, a component of the essential Smc5/6 DNA repair complex Mol Microbiol 55:1735-1750 doi:10.1111/j.1365-2958.2005.04531.x

Ju L et al. (2013) SMC6 is an essential gene in mice, but a hypomorphic mutant in the ATPase domain has a mild phenotype with a range of subtle abnormalities DNA Repair (Amst) 12:356-366 doi:10.1016/j.dnarep.2013.02.006

Kamisugi Y et al. (2012) MRE11 and RAD50, but not NBS1, are essential for gene targeting in the moss Physcomitrella patens Nucleic Acids Res 40:3496-3510

doi:10.1093/nar/gkr1272 
Kamisugi Y, Whitaker JW, Cuming AC (2016) The Transcriptional Response to DNADouble-Strand Breaks in Physcomitrella patens PLoS One 11:e0161204 doi:10.1371/journal.pone.0161204

Knight CD, Cove DJ, Cumming AC, Quatrano RS (2002) Molecular Plant Biology vol 2. Practical Approach. Oxford University Press, Oxford

Kozak J, West CE, White C, da Costa-Nunes JA, Angelis KJ (2009) Rapid repair of DNA double strand breaks in Arabidopsis thaliana is dependent on proteins involved in chromosome structure maintenance DNA Repair (Amst) 8:413-419 doi:10.1016/j.dnarep.2008.11.012

Liu YC, Vidali L (2011) Efficient polyethylene glycol (PEG) mediated transformation of the moss Physcomitrella patens J Vis Exp doi:10.3791/2560

Menand B, Calder G, Dolan L (2007) Both chloronemal and caulonemal cells expand by tip growth in the moss Physcomitrella patens J Exp Bot 58:1843-1849 doi:10.1093/jxb/erm047

Onoda F et al. (2004) SMC6 is required for MMS-induced interchromosomal and sister chromatid recombinations in Saccharomyces cerevisiae DNA Repair (Amst) 3:429439 doi:10.1016/j.dnarep.2003.12.007

Palecek JJ (2018) SMC5/6: Multifunctional Player in Replication Genes (Basel) 10 doi:10.3390/genes10010007

Pfaffl MW (2004) Quantification strategies in real-time PCR. A-Z of Quantitative PCR. International University Line, La Jolla, CA, USA

Schaefer DG et al. (2010) RAD51 loss of function abolishes gene targeting and de-represses illegitimate integration in the moss Physcomitrella patens DNA Repair 9:526-533 doi:10.1016/j.dnarep.2010.02.001

Schween G, Gorr G, Hohe A, Reski R (2003) Unique Tissue-Specific Cell Cycle in Physcomitrella Plant Biology 5:50-58 doi:10.1055/s-2003-37984

Trouiller B, Charlot F, Choinard S, Schaefer DG, Nogue F (2007) Comparison of gene targeting efficiencies in two mosses suggests that it is a conserved feature of Bryophyte transformation Biotechnol Lett 29:1591-1598 doi:10.1007/s10529-0079423-5

Trouiller B, Schaefer DG, Charlot F, Nogue F (2006) MSH2 is essential for the preservation of genome integrity and prevents homeologous recombination in the moss Physcomitrella patens Nucleic Acids Res 34:232-242 doi:10.1093/nar/gkj423

Vondrova L, Adamus M, Nociar M, Kolesar P, Oliver AW, Palecek JJ (2019) A role of the Nse4 kleisin and Nse1/Nse3 KITE subunits in the ATPase cycle of SMC5/6 bioRxiv:760678 doi:10.1101/760678 